\title{
Neuromyelitis Optica with HTLV-1 Infection: Different from Acute Progressive HAM?
}

\author{
Michiaki Koga ${ }^{1}$, Toshiyuki Takahashi ${ }^{2}$, Motoharu Kawai ${ }^{1}$, Kiyoshi Negoro ${ }^{1}$ \\ and Takashi Kanda ${ }^{1}$
}

\begin{abstract}
Acute variant of human T lymphotropic virus type 1 (HTLV-1)-associated myelopathy (HAM) has been postulated as termed "acute HAM" or "rapidly progressive HAM". However, it remains controversial whether HAM itself could cause such rapid progression. We report a patient with HTLV-1 infection, in whom the diagnosis of neuromyelitis optica (NMO) could be made based on relapsing-remitting course of opticospinal disturbance and positive anti-aquaporin-4 (AQP4) antibody. Careful testing of anti-AQP4 antibody is necessary to establish whether or not acute HAM is a clinical variant of HAM.
\end{abstract}

Key words: neuromyelitis optica, HTLV-1, acute HTLV-1-associated myelopathy, anti-aquaporin-4 antibody

(Inter Med 48: 1157-1159, 2009)

(DOI: 10.2169/internalmedicine.48.1989)

\section{Introduction}

Human T lymphotropic virus type 1 (HTLV-1)-associated myelopathy (HAM)/tropical spastic paraparesis (TSP) typically manifests with slow onset and chronic and steady progression of myelopathic deficits (1). The acute onset and rapid progression of myelopathy has been termed acute HAM or rapidly progressive HAM $(2,3)$. However, it remains controversial whether HAM itself could cause such rapid progression, since other etiologies such as multiple sclerosis (MS) are difficult to exclude. Moreover, although the presence of optic neuritis suggests the unlikeliness of HAM, some investigators reported that acute optic neuritis could occur also in HAM (4), making it difficult to differentiate HAM from MS. Here, we report a patient with HTLV1 infection who was also diagnosed with neuromyelitis optica (NMO) based on positive anti-aquaporin-4 (AQP4) antibodies.

\section{Case Report}

A 56-year-old man was admitted to a local hospital for acute progressive sensory disturbances in both legs. He had experienced a monophasic course of bilateral optic neuritis four months previously. The patient was from Miyazaki, an area endemic for HTLV-1 infection in Japan. On admission, he showed extensor plantar responses, hypesthesia below Th 10 , and severe urinary disturbances. Serological examination showed that anti-HTLV-1 antibody was positive whereas anti-nuclear antibody and anti-SS-A/SS-B antibodies were negative. Other serological data were unremarkable, although anti-AQP4 antibody was not examined. CSF analysis showed increased cell count $(208 / \mu \mathrm{L}$; monocyte $=98 \%)$, increased protein $(80 \mathrm{mg} / \mathrm{dL})$ without sugar decrease, and positive anti-HTLV-1 antibody on particle agglutination (PA) (titer, 32 or more; cut-off < 16) and Western blot analyses. T2-weighted MRI revealed a long cord lesion (six or more vertebral segments in length) with obscure gadoliniumenhancement at the thoracic cord (Fig. 1). Although multiple periventricular lesions were apparent on brain MRI (Fig. 2A), a neurologist considered the patient to be suffering from HAM. He underwent steroid-pulse therapy consisting of $1,000 \mathrm{mg} / \mathrm{day}$ intravenous methylprednisolone for three consecutive days, followed by 3MIU/day interferon- $\alpha$ (Sumiferon ${ }^{\mathrm{TM}}$ ) for one month, after which his neurological abnormalities and CSF cell count gradually lessened. The patient was followed-up by one of the authors (M.Ko.) for

${ }^{1}$ Department of Neurology and Clinical Neuroscience, Yamaguchi University Graduate School of Medicine, Ube and ${ }^{2}$ Department of Neurology, Tohoku University Graduate School of Medicine, Sendai

Received for publication December 26, 2008; Accepted for publication March 25, 2009

Correspondence to Dr. Michiaki Koga, kogamrk@yamaguchi-u.ac.jp 

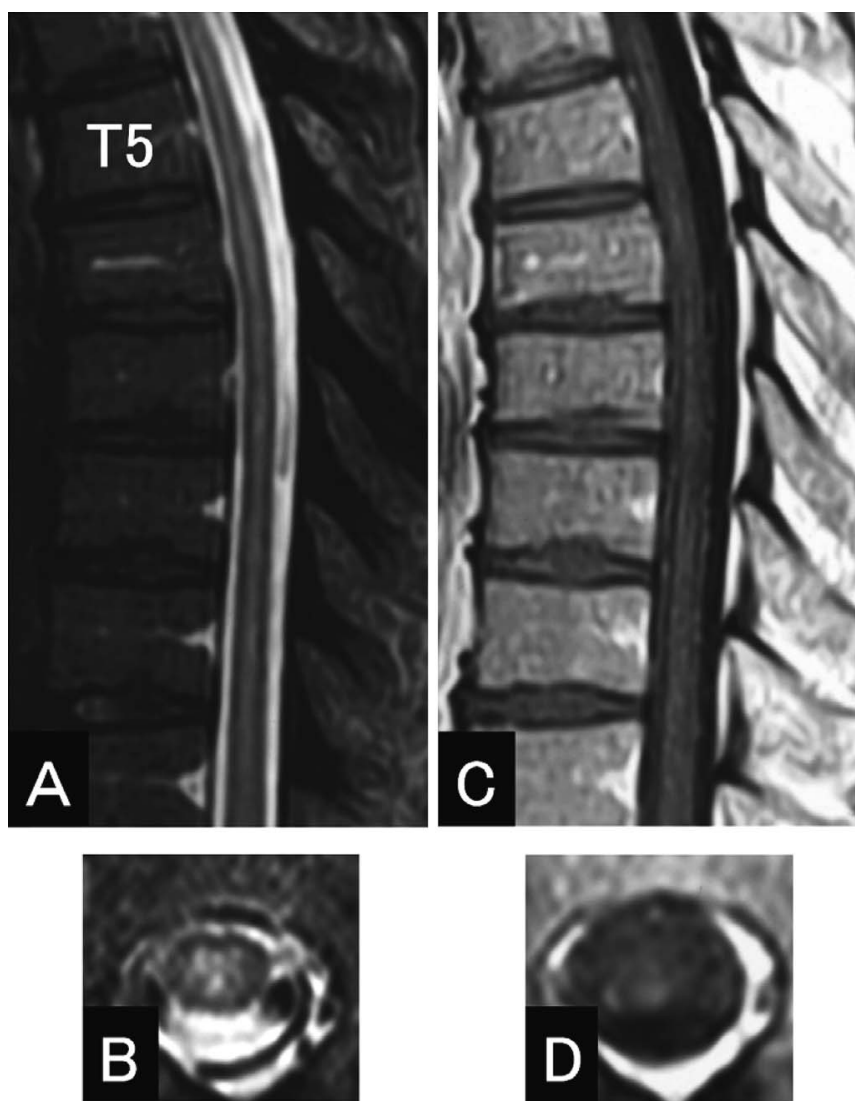

Figure 1. Thoracic cord MRI at age 56. T2-weighted images (A: sagittal image, $B$ : axial image at the $T 4$ vertebral level) showed a long cord lesion which was centrally located with obscure gadolinium-enhancement (C: sagittal image, D: axial image at the $\mathbf{T} 4$ vertebral level).

three years, and his myelopathic symptoms showed slow but steady recovery in this period.

At age 59, the patient experienced visual loss in the right eye, which was slightly resolved after steroid-pulse therapy. Five months later, he was suddenly unable to walk and was admitted to our facility. He had right blindness, complete paraplegia of both lower extremities with hypotonus and extensor plantar responses, hypesthesia below Th5, and urinary retention. Serum and CSF anti-HTLV-1 antibody titers were increased on PA (both serum and CSF titers, 256 or more; cut-off < 16) and Western blot analyses. HTLV-1 proviral DNA was also detected in both serum and CSF by PCR to amplify the $\mathrm{pX}$ region (SRL, Inc, Tokyo, Japan). Other serological data, including anti-nuclear antibody, were unremarkable, except for an extremely high anti-AQP4 IgG antibody titer $(1: 32768$; cut-off < 4) (5). Serum anti-nuclear antibody and anti-SS-A/SS-B antibodies remained negative. CSF analysis showed increased cells $(24 / \mu \mathrm{L}$; monocyte $=$ $92 \%)$, protein $(71 \mathrm{mg} / \mathrm{dL})$, and myelin basic protein $(353$ $\mathrm{ng} / \mathrm{mL}$ ), but the oligoclonal $\mathrm{IgG}$ band was negative. Brain MRI (Fig. 2B, 2C) showed increased hyperintensity lesions on FLAIR imaging, some of which were considered as periventricular ovoid lesions. Spinal MRI revealed atrophy of the thoracic cord, but neither new lesions nor abnormal enhancement, in spite of a poor study due to flow-related ar-
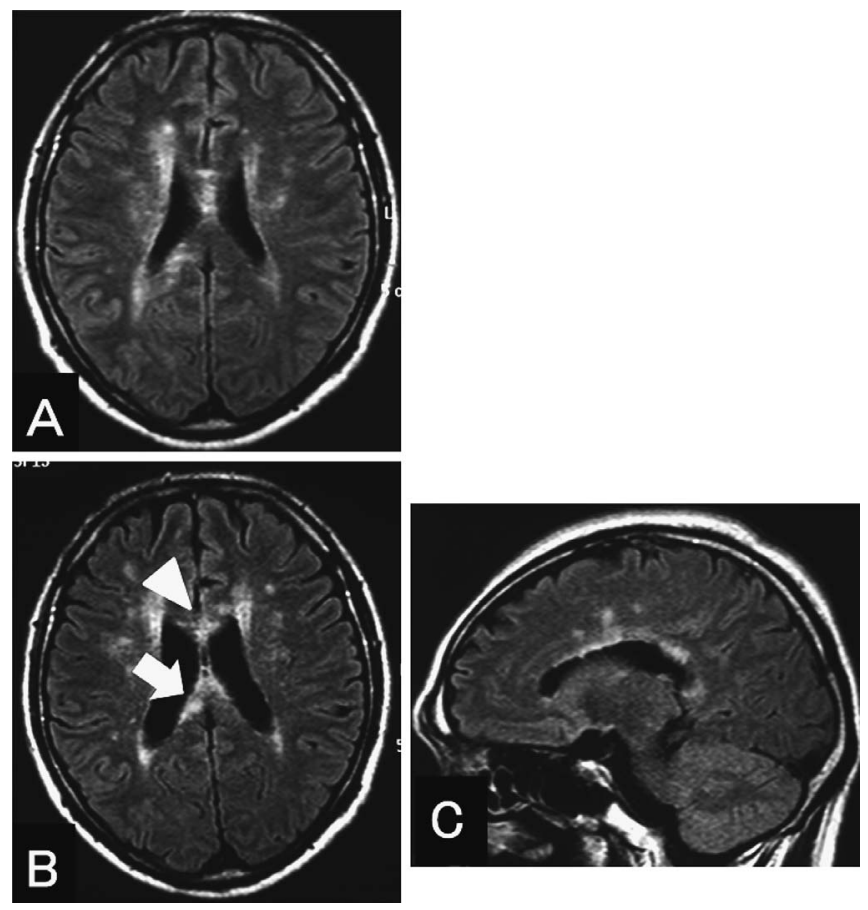

Figure 2. Brain MRI at age 56 (A) and age 59 (B and C). T2-weighted image showed multiple periventricular hyperintense areas at age 56 (A) and age 59 (B). Note that the lesions were increased, especially at the splenium (arrow) and trunk (arrowhead) of the corpus callosum at age 59. The brain also appeared slightly atrophic and ovoid lesions were present at age 59.

tifact. After three courses of steroid-pulse therapy followed by oral prednisolone $(12.5 \mathrm{mg} /$ day $)$, the patient showed very slow recovery of the myelopathic symptoms with increased muscular strength and spasticity in both legs six weeks after onset of paraplegia. Three weeks later, he was transferred to another hospital with severe residual leg weakness that left him wheelchair bound, and he required catheterization for urinary retention.

\section{Discussion}

The present patient experienced four attacks of optic neuritis or myelitis, with indications of HTLV-1 infection. Multiple and increasing brain lesions, predominantly in the corpus callosum, suggested conventional MS. In both HAM and MS, an oligoclonal IgG band in the CSF, and brain lesions on MRI may be present $(1,6)$. With acute HAM, in which myelopathic deficits rapidly progress $(2,3)$, it is difficult to differentiate MS from HAM, especially in areas endemic for HTLV-1 infection. This difficulty is largely due to the lack of laboratory markers specific for HAM or MS. Thus, the etiological position of MS with HTLV-1 antibody remains unclear (6), and it is debatable whether HTLV-1 infection is related to MS development (7).

Serum anti-AQP4 antibody was recently identified as a specific laboratory marker for NMO (8). Clinical and immunological characteristics indicate that NMO is a distinct dis- 
ease entity from MS (8). It remains controversial whether typical periventricular ovoid lesions could be present in NMO and it therefore seems impossible to rule out the possibility that the present patient suffered conventional MS. However, the severe attacks of optic neuritis and myelitis, absent oligoclonal band in CSF, and long spinal cord lesion seen in our patient are strongly suggestive of NMO. However, if anti-AQP4 antibody had not been detected in our case, it would have been unclear whether this patient had acute HAM resembling NMO or NMO with HTLV-1 infection.

Our patient was treated with interferon- $\alpha$ at first myelopathic attack, raising the possibility that positive anti-AQP4 antibody was due to the $\mathrm{B}$ cell polyclonal activation by interferon- $\alpha$ (9). However, the time interval between the interferon- $\alpha$ administration and test of serum anti-AQP4 antibody was three years, and we therefore considered that the detection of the antibody was not related to the interferon- $\alpha$ administration. Moreover, $\mathrm{T}$ cells infected with retrovirus including HTLV-1 could induce polyclonal B cell activation (10). This suggests another possibility that positive antiAQP4 antibody merely reflects the HTLV-1 infection in our patient. However, none of the $36 \mathrm{HTLV}-1$-infected patients in the report of Marignier et al. (11) had serum NMO-IgG (which binds AQP4), indicating that this possibility is very low. To our best knowledge, this is the first case of NMO with anti-AQP4 antibody with HLTV-1 infection.

It remains questionable whether the present patient had supervening NMO and HAM; however, this possibility is low, since unlike HAM, our patient did not show chronic progression. The diagnosis of HAM is based on the presence of antibodies to HTLV-1 in both serum and CSF (1), but this antibody could be derived from blood to CSF in some asymptomatic HTLV-1 carriers, indicating the low specificity of this criterion (12). Previous case reports of "acute HAM" $(2,3)$ and "HAM with relapsing cervical cord lesions" (13) did not discuss anti-AQP4 antibody, and may have been incidental NMO accompanied by typical HAM with chronic progression. Similarly, this antibody was not examined in a case of HAM with optic neuritis (4). Careful testing of anti-AQP4 antibody is necessary to establish whether or not acute HAM is a clinical variant of HAM, and to determine the appropriate therapy, since the administration of interferon might be effective for HAM but not for $\operatorname{NMO}(1,8)$.

\section{References}

1. Araujo AQC, Silva MTT. The HTLV-1 neurological complex. Lancet Neurol 5: 1068-1076, 2006.

2. Kida H, Nakagawa M, Iwasaki H, et al. A case of rapidly progressive HTLV-1-associated myelopathy (HAM). Rinsho Shinkeigaku (Clin Neurol) 37: 802-805, 1997 (in Japanese, Abstract in English).

3. Kasahata N, Shiota J, Miyazawa Y, Nakano I, Murayama S. Acute human T-lymphotropic virus type 1-associated myelopathy: a clinicopathologic study. Arch Neurol 60: 873-876, 2003.

4. Yoshida Y, Saiga T, Takahashi H, Hara A. Optic neuritis and human T-lymphotropic virus type 1-associated myelopathy: a case report. Ophthalmologica 212: 73-76, 1998.

5. Takahashi T, Fujihara K, Nakashima I, et al. Anti-aquaporin-4 antibody is involved in the pathogenesis of NMO: a study on antibody titre. Brain 130: 1235-1243, 2007.

6. Puccioni-Sohler M, Yamano Y, Rios M, et al. Differentiation of HAM/TSP from patients with multiple sclerosis infected with HTLV-1. Neurology 68: 206-213, 2007.

7. Oger J. HTLV-1 infection and the viral etiology of multiple sclerosis. J Neurol Sci 262: 100-104, 2007.

8. Wingerchuk DM, Lennon VA, Lucchinetti CF, Pittock SJ, Wein- shenker BG. The spectrum of neuromyelitis optica. Lancet Neurol 6: 805-815, 2007.

9. Le Bon A, Schiavoni G, D’Agostino G, Gresser I, Belardelli F, Tough DF. Type I interferons potently enhance humoral immunity and can promote isotype switching by stimulation dendritic cell in vivo. Immunity 14: 461-470, 2001.

10. Higuchi $M$, Nagasawa $K$, Horiuchi $T$, et al. Membrane tumor necrosis factor- $\alpha$ (TNF- $\alpha$ ) expressed on HTLV-1-infected T cells mediates a costimulatory signal for B cell activation: characterization of membrane TNF- $\alpha$. Clin Immunol Immunopathol 82: 133140, 1997.

11. Marignier R, De Sèze J, Vukusic S, et al. NMO-IgG and Devic's neuromyelitis optica: a French experience. Mult Scler 14: 440445, 2008.

12. Puccioni-Sohler M, Rios M, Carvalho SMF, et al. Diagnosis of HAM/TSP based on CSF proviral HTLV-1 DNA and HTLV-1 antibody index. Neurology 57: 725-727, 2001.

13. Umehara F, Tokunaga N, Hokezu Y, et al. Relapsing cervical cord lesions on MRI in patients with HTLV-1-associated myelopathy. Neurology 66: 289, 2006.

(C) 2009 The Japanese Society of Internal Medicine http://www.naika.or.jp/imindex.html 